\title{
Evaluating the impact of biannual school-based and community-wide treatment on urogenital schistosomiasis in Niger
}

\author{
Anna E. Phillips ${ }^{1 *}$, Zilahatou Tohon², Neerav A. Dhanani ${ }^{3}$, Boubacar Sofo², Issa Gnandou ${ }^{4}$, Boubacar Sidikou ${ }^{5}$, \\ Adamou Garba Noma², Bassirou Madougou², Oumarou Alto², Hannatou Sebangou², Kader M. Halilou², \\ Roumanatou Andia ${ }^{2}$, Amadou Garba², Alan Fenwick ${ }^{3}$ and Amina A. Hamidou ${ }^{2}$
}

\begin{abstract}
Background: The Schistosomiasis Consortium for Operational Research and Evaluation (SCORE) coordinated a fiveyear study implemented in several countries, including Niger, to provide an evidence-base for programmatic decisions regarding cost-effective approaches to preventive chemotherapy for schistosomiasis control.

Methods: This was a cluster-randomised trial investigating six possible combinations of annual or biannual community-wide treatment (CWT), school-based treatment (SBT), and holidays from mass treatment over four years. The most intense arm involved two years of annual CWT followed by 2 years of biannual CWT, while the least intensive arm involved one year of annual SBT followed by a year without treatment and two more years of annual SBT. The primary outcome of interest was prevalence and intensity of Schistosoma haematobium among 100 children aged 9-12 years sampled each year. In addition, 100 children aged 5-8 years in their first year of school and 50 adults (aged 20-55 years) were tested in the first and final fifth year of the study.

Results: In total, data were collected from 167,500 individuals across 225 villages in nine districts within the Niger River valley, Western Niger. Overall, the prevalence of S. haematobium decreased from baseline to Year 5 across all study arms. The relative reduction of prevalence was greater in biannual compared with annual treatment across all arms; however, the only significant difference was seen in areas with a high starting prevalence. Although adults were not targeted for treatment in SBT arms, a statistically significant decrease in prevalence among adults was seen in moderate prevalence areas receiving biannual (10.7\% to 4.8\%) SBT $(P<0.001)$. Adults tested in the annual SBT group also showed a decrease in prevalence between Year 1 and Year 5 (12.2\% to 11.0\%), but this difference was not significant.

Conclusions: These findings are an important consideration for schistosomiasis control programmes that are considering elimination and support the idea that scaling up the frequency of treatment rounds, particularly in areas of low prevalence, will not eliminate schistosomiasis. Interestingly, the finding that prevalence decreased among adults in SBT arms suggests that transmission in the community can be reduced, even where only school children are being treated, which could have logistical and cost-saving implications for the national control programmes.
\end{abstract}

Keywords: Schistosomiasis, Urogenital schistosomiasis, Community-wide treatment, School-based treatment, Biannual treatment, Elimination

\footnotetext{
*Correspondence: a.phillips05@imperial.ac.uk

${ }^{1}$ London Centre for Neglected Tropical Disease Research (LCNTDR),

Department of Infectious Disease Epidemiology, Imperial College

London, London, UK

Full list of author information is available at the end of the article
}

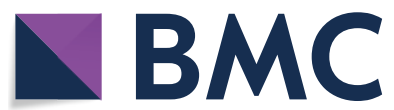

(c) The Author(s) 2020. This article is licensed under a Creative Commons Attribution 4.0 International License, which permits use, sharing, adaptation, distribution and reproduction in any medium or format, as long as you give appropriate credit to the original author(s) and the source, provide a link to the Creative Commons licence, and indicate if changes were made. The images or other third party material in this article are included in the article's Creative Commons licence, unless indicated otherwise in a credit line to the material. If material is not included in the article's Creative Commons licence and your intended use is not permitted by statutory regulation or exceeds the permitted use, you will need to obtain permission directly from the copyright holder. To view a copy of this licence, visit http://creativecommons.org/licenses/by/4.0/. The Creative Commons Public Domain Dedication waiver (http://creativecommons.org/publicdomain/zero/1.0/) applies to the data made available in this article, unless otherwise stated in a credit line to the data. 


\section{Background}

Human schistosomiasis is an acute and chronic, waterassociated parasitic disease that remains a major public health problem in sub-Saharan Africa. It represents the second most endemic parasite after malaria in these regions [1]. According to the World Health Organisation (WHO), an estimated 218 million people need preventive chemotherapy (PC) worldwide, of which $92 \%$ live in Africa [2].

It is estimated that 3.2 million people are infected with schistosomiasis in Niger [3]. Both Schistosoma haematobium (urogenital) and Schistosoma mansoni (intestinal) are endemic, but the main species is $S$. haematobium, which is distributed in all regions of the country [4]. Previously S. mansoni had a relatively marginal role; however, more recently an increase in infection has been seen in the western part of the Niger River Valley. A national programme for the control of schistosomiasis and geohelminthiasis was implemented by the Niger Ministry of Health in 2004 [5]. The control strategies used were school-based treatment (SBT) with praziquantel (PZQ) and selective chemotherapy in adults at high risk of infection, following the WHO guidelines [6].

Previously, a combined school- and community-based strategy has been shown to be effective in attaining a high coverage among school-aged children (SAC) in countries where school enrolment is low and where schools cannot serve as the exclusive drug distribution points [7-10]. There is a growing body of evidence regarding the burden of infection in adults and their potential role in sustaining transmission, which suggests a need for them to be included in treatment programmes [10-15].

Whether community-wide treatment (CWT) is appropriate depends on the local epidemiological setting and whether the goal is morbidity control or eliminating transmission [16]. Some studies, for example, have found that SBT and community-wide treatment (CWT) yielded a similar prevalence decrease $[17,18]$. There is also debate around the optimal frequency of PZQ treatment for infection and morbidity control where some studies have found that the more frequent the PC, the greater impact on parasite control [19], while others have shown that a single dose of $\mathrm{PZQ}$ results in sustained low transmission of $S$. haematobium for 2 years [20]. Considering the controversy around the optimal approach to drug-based control of schistosomiasis, the Schistosomiasis Consortium for Operational Research and Evaluation (SCORE) was established in 2008 to address this and other questions of practical significance. An important part of SCORE's portfolio was multi-country field studies, which included Niger, that have evaluated the impact of alternative approaches to
PC through CWT, SBT, and years without mass drug administration (MDA) [21].

The SCORE protocol and baseline characteristics of study populations have been described elsewhere [22, 23]. In brief, SCORE projects include "Gaining control of schistosomiasis" studies, evaluating PC in communities with high prevalence of schistosomiasis, and "Sustaining control of schistosomiasis" studies, examining PC in areas of moderate schistosomiasis prevalence [21].

In 2011, SCORE began supporting Niger to conduct both "Gaining" and "Sustaining" S. haematobium control studies in two separate parts of the Niger River Valley. A simple random allocation approach was supposed to have been used to assign all eligible villages to study arms. Instead, during the selection of the field sites, Niger geographically clustered groups of villages into study arms. Following Year 2 of the study, when this was recognised, the Niger study was redesigned to compare annual versus biannual SBT or CWT. An additional deviation from the SCORE protocol included replacing the treatment 'holiday' years with a test-and-refer strategy, whereby mass drug administration was not given on these years, but individuals were tested and those found to be positive were referred to health centres for treatment.

The primary research question presented here is which PC strategy provided the greatest reduction in prevalence and intensity of S. haematobium infection among 9-12 year-olds after four years of intervention. In addition, the impact of treatment on first-year students and adults was also assessed.

\section{Methods}

Study design

SCORE implemented a parallel cluster-randomised intervention trial that includes six study arms for the "Gaining" control study and three arms for the "Sustaining" control study [21]. The primary outcome of interest was the prevalence and intensity among 9-12 year-old children following four years of MDA using different approaches. In Niger, the "Gaining" study was to be implemented in an area that historically had high levels of infection, and the "Sustaining" study in a nearby area expected to have lower transmission.

The original design relied on randomisation so that starting prevalence would be roughly equivalent in different study arms. However, because of the way in which the Niger study clustered villages, the starting prevalence were markedly different in different arms, so valid comparisons between arms and comparison with other SCORE studies could not be made. Therefore, in 2013 (before Year 3 of the study) the Niger study was re-designed to evaluate the impact of either SBT or CWT twice a year (biannual) versus annual treatment 
with PZQ. Thus, the communities received a variable sequence of SBT, CWT or test/refer for treatment strategies for the first two years, and then once or twice-yearly treatment for the final two treatment years, with the final round of data collection done 12 months after the final (Year 4) treatment round. The reformatted study design, upon which the following analyses are based, is summarised in Fig. 1.

The 75 communities found eligible with $10-24 \%$ prevalence initially received either SBT in Years 1 and 2, or SBT in Year 1 and were tested and referred for treatment in Year 2. Before Year 3 these were combined and re-randomised into Arm A1, which received SBT once a year in Years 3 and 4, or Arm A2, which received SBT twice a year in Years 3 and 4. Of the communities found eligible with $\geq 25 \%, 75$ received either SBT in Years 1 and 2, or SBT in Year 1 and were tested and referred for treatment in Year 2. Before Year 3 these were combined and re-randomised into Arm B1, which received SBT once a year in Years 3 and 4, or Arm B2, which received SBT twice a year in Years 3 and 4 . The other 75 communities found eligible with $\geq 25 \%$ received CWT in Years 1 and 2 and before Year 3 were re-randomised into Arm C1, which received CWT once in Years 3 and 4 and Arm C2 which received CWT twice a year in Years 3 and 4 (Fig. 1).

The number of villages per arm and number of 9-12 year-old students tested per village in the original study design were based on sample size calculations [21]. Based on these calculations, a village's prevalence and intensity of S. haematobium infections were monitored each year among a random sample of 100 school children aged 9-12 years. A total of 225 villages were enrolled, with 22,500 children aged 9-12 years tested each year. In addition, systematic random sampling was used in each village to select a further 100 first-year students (aged 5-8 years) in all 225 villages at the first and fifth years only. A convenience sample of 50 adults (aged 22-50 years) were sampled in the first and last year of the "Gaining" study.

Communities were only eligible to participate in the study if they had a primary school with at least 100 school children aged 9-12 years and if they met the prevalence criteria for either "Gaining" or "Sustaining" control studies. Prevalence was assessed by testing fifty children aged 13-14 years in each potential study community using reagent Hemastix ${ }^{\circledR}$ testing for microhaematuria on a single midday urine sample [24]. Trace results were considered positive per manufacturer instructions. Communities in the area selected for the "Gaining" studies were eligible if they had high prevalence ( $\geq 25 \%$ ), and for "Sustaining" studies if they had moderate prevalence (10-24\%) among 13-14 year-olds in the eligibility assessment (the 13-14 year-old age group was used to assess eligibility because of the need to treat children testing positive, which would have affected baseline study results).

The 150 communities found eligible for "Gaining" were initially assigned to one of six arms of the original study design and the 75 villages eligible for "Sustaining" were allocated to one of three study arms in the original study

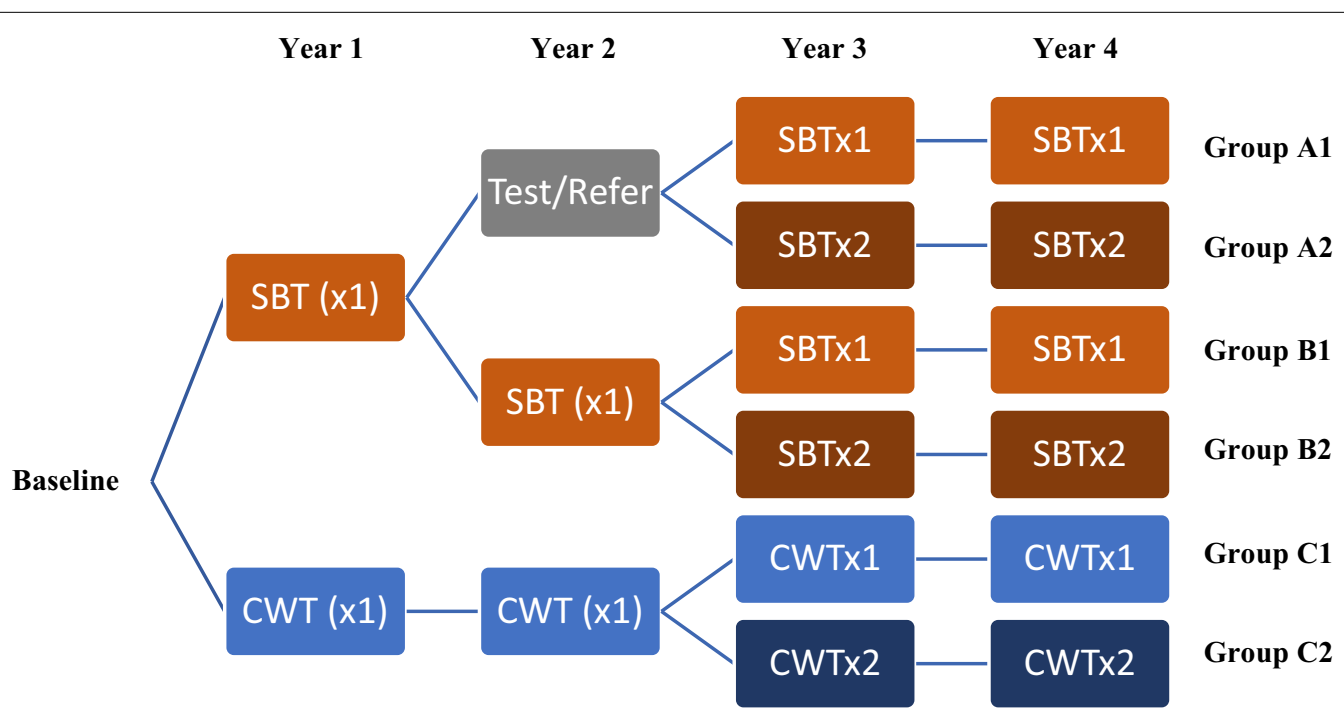

Fig. 1 Niger SCORE study design combining "Gaining" and "Sustaining" studies. There are six study arms, where by each arm received a four-year treatment strategy with varying combinations of annual school-based treatment (SBTx1), biannual school-based treatment (SBTx2), annual community-wide treatment (CWTX1), biannual community-wide treatment (CWTX2), and "Test \& Treat" (i.e. where test and referral for treatment): Arm 1: SBTx1-Test \&Treat-SBTx1-SBTx1; Arm 2: SBTx1-Test \& Treat-SBTx2-SBTx2; Arm 3: Annual SBT; Arm 4: SBTx1-SBTx1- SBTx2-SBTx2; Arm 5: Annual CWT; Arm 6: Biannual CWT 
design. When the study was re-designed, each arm's villages were randomly assigned to once- $v s$ twice-a-year treatment using a computer-based randomisation. Thus, communities received a variable sequence of $\mathrm{CWT}$, $\mathrm{SBT}$, or test/refer for treatment strategies for the first two years, and then once or twice-yearly treatment for the final 2 years, with the final round of data collection carried out 12 months after the final (Year 4) treatment round in Year 4.

\section{Study area and population}

The study was conducted in the region of Tillabery (Kollo, Say, Tera, Filingué and Tillabery districts), Dosso (Loga district) and Niamey (districts 1-3) in Western Niger along the Niger River Valley (Fig. 2). The climate is characterised by a short rainy season from June to September and a long dry season from October to May. Eight districts are only endemic to $S$. haematobium, but the district of Tillabery is endemic to S. haematobium and to a lesser extent $S$. mansoni. The main sites for transmission are irrigation canals, rice paddies and temporary water bodies that fill up during the rainy season. The main occupations of the population are agriculture and livestock. Millet is grown during the rainy season, while rice is grown in the Niger River valley twice a year. PC has been implemented in the study area since 2004 by the national schistosomiasis control programme. Mass treatment with praziquantel has been provided more than eight times with acceptable coverage rates [5]. Although schooling is compulsory in principle for ages 7-15 years-old for a period of eight years, there is only about a $25 \%$ school attendance by primary schoolage children, and even fewer 12-17 year-olds continue to secondary school [25]. Sanitation in the study area is also low; over $20 \%$ of deaths in Niger can be attributed to poor sanitation and hygiene [26]. While Niger has one of the lowest sanitation coverage rates in the world, access to improved sanitation has increased over the last ten years. However, Niger has one of the highest population growth rates in the world, which has likely blunted the impact of some of the progress being made $[27,28]$.

\section{Study participants}

The 9-12 year-old children were selected based on a list of students provided by the teacher with the name, sex and age of each child in the class, with younger children being selected first and recruitment continuing until the target number was achieved. In schools where less than 100 children were available, community leaders encouraged parents of non-school attending children to volunteer and enrol in the survey. All children were given a container in which to provide a single urine sample collected between 10:00 and 14:00 h. A similar approach was used to select first-year students who were 5-8 years-old for testing in Years 1 and 5. Convenience samples of adults were recruited to participate in the study by presenting at the school on the day of data collection on a voluntary basis as a convenience sample.

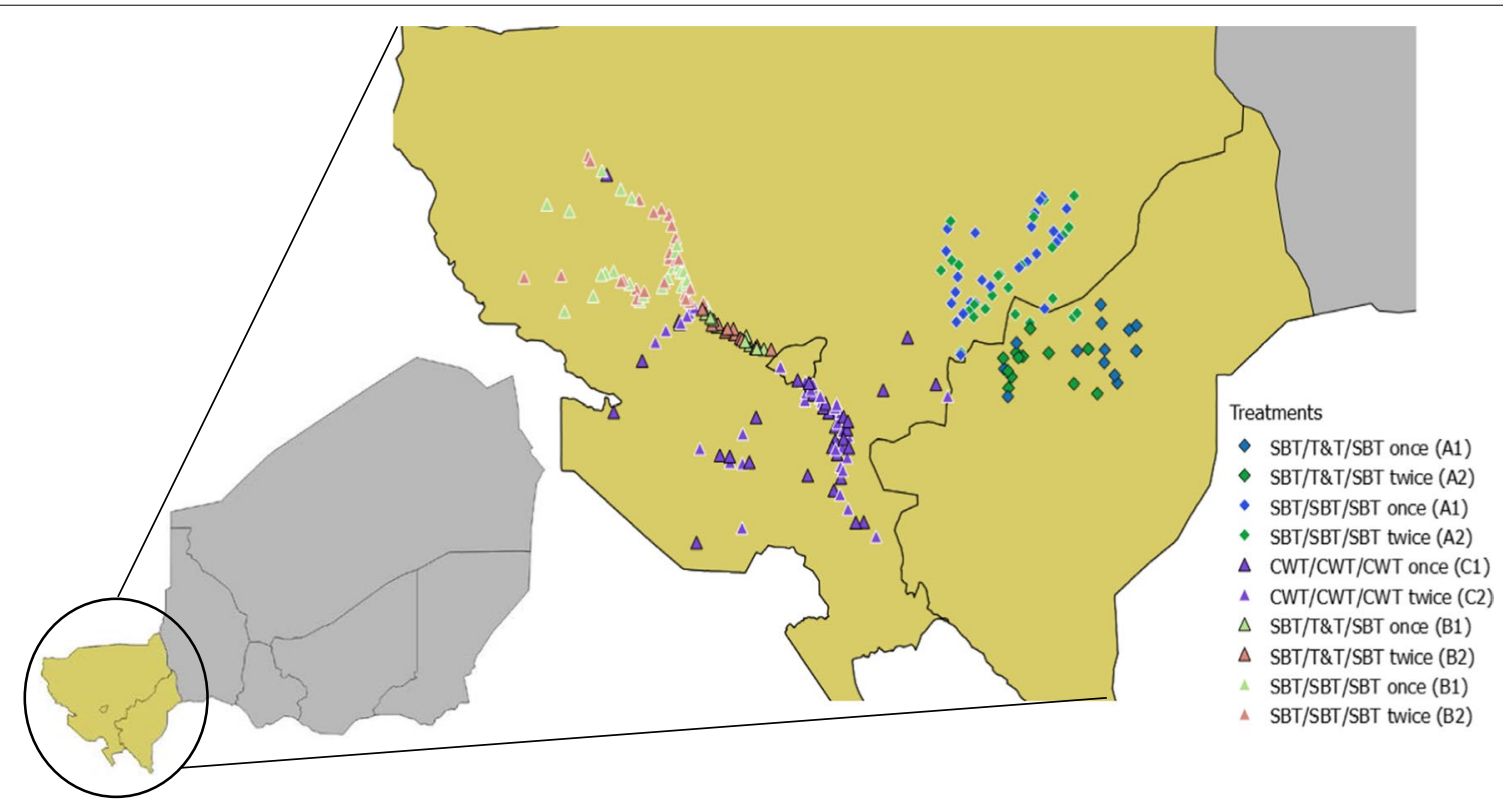

Fig. 2 Map of the study zone. The site of each village has been colour-coded on the map according to the study arm 


\section{Urine examination}

A single urine was collected from each individual in the study for assessment of parasitological outcomes, two filtrations were carried out on two $10 \mathrm{ml}$ aliquots of each urine sample using a $\mathrm{Nytrel}^{\circledR}$ mesh according to the method of Plouvier et al. [29]. One filtration was examined immediately in the school, and the second filtration by another technician in the national schistosomiasis laboratory. Both filters were coloured with lugol and S. haematobium eggs were counted under a light microscope. Intensity of infection was defined as negative (no eggs found), light (1-49 eggs/10 ml), and heavy ( $\geq 50 \mathrm{eggs} / 10$ $\mathrm{ml}$ ) infection [6]. Number of eggs counted was capped at 1000 eggs $/ 10 \mathrm{ml}$. Where urine volume was less than 10 $\mathrm{ml}$, the volume of urine was measured, and the number of eggs recalculated per $10 \mathrm{ml}$. The mean number of eggs of child was calculated as the arithmetic mean of the two filtrations taken from a single urine sample, including both egg positive and negative. A person was deemed egg-positive if one or more eggs were found in any of the slides examined.

\section{Treatment}

Prior to treatment, community sensitisation was carried out using "public criers" and local radios to inform and mobilise the population to participate. In SBT villages, children were treated with a single dose of PZQ regardless of parasitological results [21], unless they met the WHO treatment exclusion criteria [6]. In villages receiving CWT, the entire eligible population were treated, with only children under 5 years of age or under $94 \mathrm{~cm}$ in height excluded [6]. In test/refer for treatment villages, children who tested positive were referred to health centres for treatment. The dose of PZQ for children was determined using a WHO dose pole [30].

In CWT, drugs were distributed door-to-door by trained community drug distributors (CDDs). All CDDs were selected by the community and trained by the local health facility nurse prior to each treatment campaign. In SBT, PZQ was administered by trained teachers to all primary school-aged children, both enrolled and non-enrolled, regardless of infection status. Training and supervision for both CWT and SBT were provided each year prior to the MDA by local health workers. The importance of directly observed therapy was emphasised at training, which was closely monitored particularly in schools as the survey teams collected urine samples on the same day (prior) to treatment. In the communities, the CDD were spot checked to ensure that compliance was adhered to. Coverage was estimated using the national population census estimates as a denominator. Where coverage was less than $75 \%$, additional "mop-up" efforts were made to increase the coverage. Treatment numbers were recorded on registers and collected at the end of the MDA by the health workers and transmitted to the national control programme. Any secondary adverse effects were reported.

\section{Data management and analysis}

All demographic and laboratory data were entered on paper forms and double-entered into Microsoft Excel. Data cleaning and management was carried out by RISEAL Niger. Data analysis was performed using R version 3.2 [31]. The parasitological results were averaged across each village to produce a village level prevalence and village level prevalence of heavy infected, respectively. 95\% confidence intervals were calculated for village- and study arm-level prevalence values. Prevalence of infection and heavy infection were calculated as arithmetic means of the infection categories, aggregated by the relevant factors (e.g. age, sex). The arithmetic mean of infection intensity was calculated using both egg-positive and negative, aggregated by grouping factors (village, study arm, and sex). Although mean intensity might have been underestimated as egg counts were capped at 1000 eggs $/ 10 \mathrm{ml}$, this was done consistently over the years. Therefore, the trend in intensity change is believed to be valid. The egg reduction rate was calculated as the reduction in the intensity of infection assessed indirectly, using egg count via the following formula: \% egg reduction $=100 \times(1-($ Arithmetic mean of eggs $/ 10 \mathrm{ml}$ urine after treatment in Year 5 / Arithmetic mean of eggs $/ 10 \mathrm{ml}$ urine at baseline)).

Village-level prevalence was calculated as the percent of children having at least one egg detected by urine filtration. Once and twice-yearly treatment were compared using a generalised linear mixed model (GLMM). The models were run on three subsets of the data, the 9-12 year-old cross-section, 20-55 year-olds (adult) and 5-8 year-olds. The lme 4 package was used in $\mathrm{R}$ and the function glmer to model the difference in the chance of being infected at the end of the study between treatment groups. The parameter being modelled was a binary factor to indicate infection status $(0=$ not infected, $1=$ infected). Treatment group, sex and age were included as fixed effects and village was included as a random effect. To avoid issues of multiple testing we focused on group comparisons. In Years 3 and 4: Group A in low prevalence areas received SBT once a year (A1), and twice a year (A2); Group B in high prevalence areas receiving SBT once a year (B1), and twice a year (B2); and Group C in high prevalence areas receiving CWT once a year $(\mathrm{C} 1)$, and twice a year (C2). For Group $C$, the prevalence at the start of the study was significantly different between villages in $\mathrm{C} 1$ and those in $\mathrm{C} 2$. Therefore, baseline data were included in the analysis and the model was adjusted to 
include study year and an interaction term between study year and treatment group as fixed effects.

\section{Results \\ Eligibility survey}

The eligibility survey was conducted between October 2010 and January 2011. In total 19,990 children aged 13-14 years were randomly selected from 348 villages (where 50 children were randomly selected and tested in each school) and screened for haematuria using urine dipsticks. Overall, 150 villages were screened in moderate endemicity areas for the "Sustaining" control study, of which 75 met the criteria (haematuria prevalence of $10-24 \%)$. A total of 248 villages were screened for the high prevalence "Gaining" control study, of which 150 met the criteria (haematuria prevalence $\geq 25 \%$ ).

\section{Treatment coverage}

Drug coverage was defined as the proportion of individuals who were directly observed to have ingested the PZQ. CWT coverage was determined by the total population treated using estimated projections of the denominator of the whole population based on a population census carried out in 2011, which is also used by the National Schistosomiasis and STH control programme to calculate treatment coverage. The SBT coverage was determined by the percentage of all school-aged children (5-12 years-old) that were treated as a proportion of school-aged children estimated in the population census, and therefore included children not enrolled in school. Treatment coverage is summarised in Table 1.

The nominal treatment coverage stipulated by the study protocol was coverage of more than $75 \%$ for both SBT and CWT. Overall, there was extremely high coverage across all arms, this was expected the communities enrolled in this community are rural and isolated. However, in some Groups there is reported coverage above $100 \%$. This could be explained by an inaccurate denominator, migration into the community for treatment, and treatment of adults receiving treatment during the SBT. When coverage was included into the multivariate model looking at impact on changes of prevalence over time, there was no clear trend or significant relationship with impact on infection.

\section{Summary sample characteristics by study year for all individuals examined}

Data were collected in the dry season between October and February each year from 2011 until 2015 across nine districts in the Niger River Valley. The aim was to sample 167,500 individuals from a total of 225 villages over the five years. In total 166,811 individuals were tested.
Table 2 shows the sample characteristics by study year, by age group. Overall, nearly half of individuals sampled were female consistently across all study years and age groups.

\section{9-12 year-olds cross-section}

Over five years, 108,231 independent observations were taken from children aged 9-12 years and the prevalence and intensity of the infections in this group were designated as the primary outcome of the study. Table 3 shows the change in S. haematobium infection between Baseline and Year 5 in low prevalence areas treated once and twice a year with SBT only (Group A), SBT in high prevalence areas (Group B), and CWT in high prevalence areas (Group C). As expected, the relative reduction of prevalence is greater in biannual treatment compared with annual MDA across all study arms. The biggest difference is seen in arms starting in high prevalence areas, $\mathrm{C} 2$ and $\mathrm{B} 2$ receiving biannual treatment where absolute difference in prevalence between Year 5 and baseline was $12.9 \%$ and $9.1 \%$, respectively. The egg reduction rate follows the same pattern as the prevalence rate, again with a decrease in intensity of infection at both the village-and individual-level across all study arms. Group B shows the largest difference in the egg reduction rate between the treatment strategies $(72.0 \%$ at baseline to $12.0 \%$ in Year $5)$.

Figure 3 shows the change in overall prevalence of $S$. haematobium infection over time from the start of the project at baseline to the final Year 5 by treatment group. The starting prevalence of infected individuals in Group $\mathrm{A}$ is low at baseline in both $\mathrm{A} 1$ and $\mathrm{A} 2$ (3.7\% and 4.6\%, respectively) falling to nearly $0 \%$ in Year 5 . The GEE shows no statistical difference between the arms at Year 5 for Group A. Group B had a higher prevalence at baseline in both B1 and B2 (24.5\% and 22.5\%, respectively), with a reduction in Year 5 (23.2\% and $13.4 \%$, respectively). The GEE showed that the difference in prevalence within Group B at Year 5 was significant with B2 being lower than B1 $(P<0.037)$. Group C had a prevalence of $14.3 \%$ in $\mathrm{C} 1$ and $23.4 \%$ in $\mathrm{C} 2$ with prevalence in Year 5 being $11.5 \%$ and $10.5 \%$, respectively. There was no statistically significant difference between $\mathrm{C} 1$ and $\mathrm{C} 2$ at Year 5 .

Figure 4 shows the change in overall prevalence and mean intensity of $S$. haematobium at each time point from the start of the project at baseline to the final Year 5 of the study. In all arms, there was a decrease in prevalence and mean intensity over time. In Groups B and C, however, there was a peak in prevalence and intensity of infection in Year 3.

Prevalence by sex showed a higher prevalence of infection among boys than girls, which was significant in Group B only $(P<0.001)$ (Table 4$)$. Response to treatment 
Table 1 Treatment coverage by Group over time

\begin{tabular}{|c|c|c|c|c|c|c|c|}
\hline Year (Y) & Study group & SAC treated & SAC total & $\begin{array}{l}\text { Mean \% SAC } \\
\text { treated }^{\mathrm{a}}\end{array}$ & $\begin{array}{l}\text { Total population } \\
\text { treated }\end{array}$ & $\begin{array}{l}\text { Total population } \\
\text { eligible for treatment }\end{array}$ & $\begin{array}{l}\% \text { total } \\
\text { population } \\
\text { treated }\end{array}$ \\
\hline \multirow[t]{6}{*}{ Y1 } & $\mathrm{A} 1$ & 16,563 & 20,267 & 82 & & & \\
\hline & $A 2$ & 18,986 & 18,809 & 101 & & & \\
\hline & B1 & 20,382 & 24,670 & 83 & & & \\
\hline & B2 & 25,281 & 30,373 & 83 & & & \\
\hline & $\mathrm{C} 1$ & 22,479 & 17,598 & 128 & 43,600 & 58,648 & 74 \\
\hline & C2 & 26,373 & 25,133 & 105 & 57,058 & 83,772 & 68 \\
\hline \multirow[t]{6}{*}{ Y2 } & $\mathrm{A} 1$ & 15,053 & 13,425 & 112 & & & \\
\hline & $A 2$ & 13,553 & 14,309 & 95 & & & \\
\hline & B1 & 19,322 & 17,378 & 111 & & & \\
\hline & B2 & 16,345 & 17,227 & 95 & & & \\
\hline & $\mathrm{C} 1$ & 23,512 & 18,123 & 130 & 47,031 & 60,410 & 78 \\
\hline & C2 & 33,735 & 25,883 & 130 & 66,671 & 86,287 & 77 \\
\hline \multirow[t]{6}{*}{ Y3 Treatment 1} & $\mathrm{~A} 1$ & 23,281 & 23,021 & 101 & & & \\
\hline & $A 2$ & 25,174 & 23,586 & 107 & & & \\
\hline & B1 & 28,406 & 22,999 & 124 & & & \\
\hline & B2 & 33,231 & 27,781 & 120 & & & \\
\hline & $\mathrm{C} 1$ & 26,306 & 19,663 & 134 & 51,598 & 52,434 & 98 \\
\hline & C2 & 39,311 & 24,415 & 161 & 74,558 & 65,106 & 115 \\
\hline \multirow[t]{6}{*}{ Y3 Treatment 2} & $\mathrm{~A} 1$ & 0 & 23,021 & & & & \\
\hline & $\mathrm{A} 2$ & 28,203 & 23,586 & 120 & & & \\
\hline & B1 & 0 & 22,999 & & & & \\
\hline & B2 & 25,274 & 27,781 & 91 & & & \\
\hline & $\mathrm{C} 1$ & 0 & 19,663 & & & 52,434 & \\
\hline & C2 & 33,003 & 24,415 & 135 & 61,182 & 65,106 & 94 \\
\hline \multirow[t]{6}{*}{ Y4 Treatment 1} & $\mathrm{~A} 1$ & 20,708 & 25,368 & 82 & & & \\
\hline & $A 2$ & 18,178 & 21,528 & 85 & & & \\
\hline & B1 & 27,316 & 29,813 & 92 & & & \\
\hline & B2 & 29,202 & 33,822 & 86 & & & \\
\hline & $\mathrm{C} 1$ & 22,664 & 19,042 & 120 & 42,464 & 50,761 & 84 \\
\hline & C2 & 33,861 & 25,866 & 131 & 55,831 & 68,967 & 81 \\
\hline \multirow[t]{6}{*}{ Y4 Treatment 2} & $\mathrm{~A} 1$ & 0 & 25,368 & & & & \\
\hline & $A 2$ & 16,309 & 21,528 & 76 & & & \\
\hline & B1 & 0 & 29,813 & & & & \\
\hline & B2 & 26,247 & 33,822 & 78 & & & \\
\hline & $\mathrm{C} 1$ & 0 & 19,042 & & & 50,761 & \\
\hline & C2 & 29,290 & 25,866 & 113 & 54,539 & 68,967 & 79 \\
\hline
\end{tabular}

${ }^{a}$ Arithmetic mean weighted by village SAC population

was similar by sex, with both males and females showing a reduction of prevalence over time from baseline to Year 5 in most groups (Fig. 5). There was, however, a peak in prevalence of $S$. haematobium infection at Year 3 in both boys and girls in Groups B and C.

The adjusted ORs of $S$. haematobium infection from the GLMM multivariate logistic regression models are presented in Table 4. Significance testing to assess whether the prevalence of S. haematobium varied over time between Groups showed that sex and age had a significant effect on infection status (Table 3). The model shows that individuals who are male are almost 1.5 times more likely than females to be infected in Group B (AOR: $1.3, P<0.001$ ). Children of 9 years old were more likely to be infected than older children in the 9-12 year-olds cross-section. This was significant in Group A with 10 year-olds (AOR: 0.34, $P=0.052$ ); in Group B with 11 (AOR: $0.75, P=0.011$ ) and 12 year-olds (AOR: $0.62, P<$ 
Table 2 Summary of sample characteristics by study year for all individuals examined (5-8 years-old, 9-12 years-old, and adults)

\begin{tabular}{|c|c|c|c|c|c|c|}
\hline Cross-section & Variable & Baseline (2011) & Year 2 (2012) & Year 3 (2013) & Year 4 (2014) & Year 5 (2015) \\
\hline \multirow[t]{5}{*}{$5-8$ years } & No. of individuals sampled & 20,220 & & & & 22,364 \\
\hline & Proportion female (\%) & $9328(46.1)$ & & & & $10,227(45.8)$ \\
\hline & No. infected with S. haematobium (\%) & $3474(17.2)$ & & & & $2134(9.54)$ \\
\hline & No. with heavy intensity of infection (\%) & $391(1.93)$ & & & & $239(1.07)$ \\
\hline & Arithmetic mean egg count ${ }^{\mathrm{a}}$ & 1.08 & & & & 0.62 \\
\hline \multirow[t]{5}{*}{$9-12$ years } & No. of individuals sampled & 20,931 & 21,833 & 21,620 & 21,715 & 22,132 \\
\hline & Proportion female (\%) & $9438(45.1)$ & $9697(44.4)$ & $9771(45.2)$ & $9943(45.8)$ & $10,28146.5)$ \\
\hline & No. infected with S. haematobium (\%) & $3314(15.8)$ & $2089(9.57)$ & 3809 (17.6) & $1794(8.26)$ & $2190(9.89)$ \\
\hline & No. with heavy intensity of infection (\%) & $276(1.32)$ & $99(0.45)$ & $292(1.35)$ & $146(0.67)$ & $146(0.66)$ \\
\hline & Arithmetic mean egg count ${ }^{a}$ & 3.05 & 1.27 & 3.27 & 1.42 & 1.45 \\
\hline \multirow[t]{5}{*}{ Adults ( $20-55$ years) } & No. of individuals sampled & 7041 & & & & 9,955 \\
\hline & Proportion female (\%) & $3966(56.3)$ & & & & $4789(48.1)$ \\
\hline & No. infected with S. haematobium (\%) & $793(11.3)$ & & & & $493(4.95)$ \\
\hline & No. with heavy intensity of infection (\%) & $35(0.50)$ & & & & $28(0.28)$ \\
\hline & Arithmetic mean egg count ${ }^{\mathrm{a}}$ & 4.61 & & & & 2.05 \\
\hline
\end{tabular}

a The mean egg count among all tested subjects (including those with zero egg counts), which is a measure of community level contamination potential

Table 3 Summary of S. haematobium infection by study arm from baseline to Year 5 (9-12 years-old cross-section only)

\begin{tabular}{|c|c|c|c|c|c|c|c|}
\hline \multirow[t]{2}{*}{ Variable } & \multicolumn{6}{|l|}{ Group $^{a}$} & \multirow[t]{2}{*}{ Total } \\
\hline & A1 & $\mathrm{A} 2$ & B1 & B2 & $\mathrm{C} 1$ & $\mathrm{C} 2$ & \\
\hline No. of villages sampled & 38 & 37 & 37 & 38 & 37 & 38 & 225 \\
\hline No. tested at baseline & 3461 & 3221 & 3659 & 3671 & 3357 & 3562 & 20,931 \\
\hline No. infected at baseline & 129 & 148 & 898 & 825 & 480 & 834 & 3314 \\
\hline Prevalence at baseline (\%) & 3.7 & 4.6 & 24.5 & 22.5 & 14.3 & 23.4 & 15.8 \\
\hline Prevalence of heavy infection at baseline (\%) & 0.29 & 0.28 & 1.86 & 1.93 & 0.86 & 2.50 & 1.29 \\
\hline No. tested at Year 5 & 3710 & 3648 & 3621 & 3740 & 3689 & 3724 & 22,132 \\
\hline No. infected at Year 5 & 22 & 9 & 839 & 503 & 425 & 392 & 2190 \\
\hline Prevalence at Year 5 (\%) & 0.6 & 0.2 & 23.2 & 13.4 & 11.5 & 10.5 & 9.90 \\
\hline Prevalence of heavy infection at Year 5 (\%) & 0.13 & 0.03 & 1.77 & 0.64 & 0.57 & 0.83 & 0.66 \\
\hline Absolute difference in prevalence at Year 5 and baseline & -3.1 & -4.4 & -1.3 & -9.1 & -2.8 & -12.9 & -5.90 \\
\hline Relative difference in prevalence at Year 5 and baseline (\% change) & -83.8 & -95.7 & -5.3 & -40.4 & -19.6 & -55.1 & -37.3 \\
\hline Village-level arithmetic mean infection intensity at baseline $\mathrm{e}^{\mathrm{b}}$ & 0.77 & 0.52 & 4.11 & 5.1 & 1.78 & 5.37 & 2.94 \\
\hline Village-level arithmetic mean infection intensity at Year $5^{\mathrm{b}}$ & 0.14 & 0.05 & 3.62 & 1.39 & 1.37 & 2.18 & 1.46 \\
\hline Egg reduction rate (1-Year 5 intensity/baseline) & 0.82 & 0.9 & 0.12 & 0.73 & 0.23 & 0.59 & 0.57 \\
\hline Individual-level arithmetic mean infection intensity at baseline ${ }^{c}$ & 0.84 & 0.58 & 4.05 & 4.91 & 1.85 & 5.64 & 2.98 \\
\hline Individual-level arithmetic mean infection intensity at Year $5^{c}$ & 0.14 & 0.05 & 3.61 & 1.38 & 1.37 & 2.2 & 1.46 \\
\hline
\end{tabular}

a Study arms: Low prevalence areas receiving SBT once a year (A1), and twice a year (A2); high prevalence areas receiving SBT once a year (B1), and twice a year (B2); and high prevalence areas receiving CWT once a year (C1), and twice a year (C2)

b Village-level intensity: This is the mean egg count for all tested 9-12 year-old subjects (including those with zero egg counts), which is a measure of community level contamination potential

c Individual-level intensity: This is the mean egg count among egg-positive 9-12 year-old subjects, which is an estimate of the intensity of infection among known active cases

0.001 ) and Group $C$ with 12 year-olds only (AOR: 0.74, $P$ $=0.019$ ). The impact of biannual versus annual treatment was also assessed with the GLMM model, where twice yearly treatment had a significantly greater impact in reducing prevalence of infection in Group B only (AOR: $0.43, P=0.037)$. 


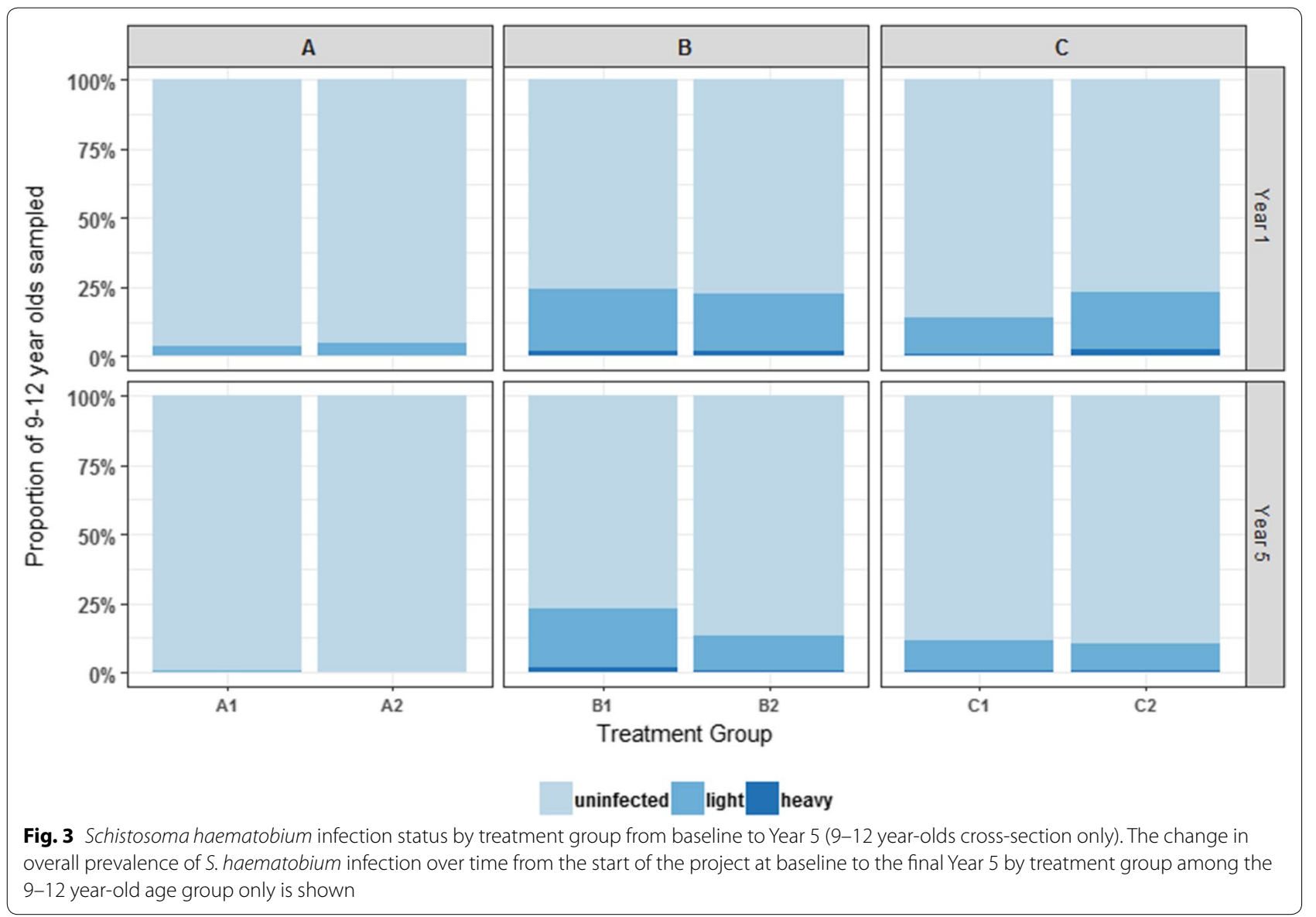

\section{First-year students and adult cross-sections}

In Year 1 and 5 of the study, an additional 42,584 and 16,996 observations were recorded for first-year students (aged 5-8 years) and adults (aged 20-55 years), respectively (Table 1 ). Overall, prevalence and intensity of infection decreased in both cross-sections over time. At baseline, examination revealed that prevalence was higher among first-year students (17.2\%) compared to $11.3 \%$ of adults infected with $S$. haematobium, which fell to $9.54 \%$ and $4.95 \%$ in Year 5, respectively. The proportion of heavily infected individuals was low even at baseline, with $1.93 \%$ first-year students and $0.5 \%$ of adults, decreasing to $1.07 \%$ and $0.28 \%$ heavy infection in Year 5, respectively (Table 1).

When separated by treatment group, prevalence, and intensity (with exception of Group C2 in adults) reduced in both adults and first year students across all arms from baseline to Year 5 (Fig. 6). Of interest is the reduction in adult prevalence that is statistically significant in both B1 (12.2\% to $11 \%)$ and B2 (10.7\% to $4.8 \%)$ arms where SBT was given only $(P<0.001)$. This would suggest that SBT alone has an impact on disease transmission.

\section{Discussion}

The SCORE project commenced in Niger with the goal of providing an evidence base for programmatic decisions on how best to control urogenital schistosomiasis, and ultimately eliminate it as a public health problem. The initial aim of this study was to examine the impact of SBT, CWT and treatment holidays for the control of S. haematobium in villages with both high $(\geq 21 \%)$ and moderate (10-24\%) prevalence. In Year 3 the study was redesigned, however, to compare annual versus biannual treatment delivered by SBT or CWT.

The primary research question presented here is which PC strategy provided the greatest reduction in prevalence and intensity of $S$. haematobium infection among 9-12 year-olds after four years of intervention in the Niger River Valley. Biannual SBT treatment was found to be significantly more effective for reducing active schistosome infection than annual SBT in high prevalence areas (Arm B1 vs B2). There was no significant effect of treatment frequency, however, in areas with a low starting prevalence (Arm A1 vs A2), or in higher prevalence areas receiving CWT (Arm C1 vs C2). 

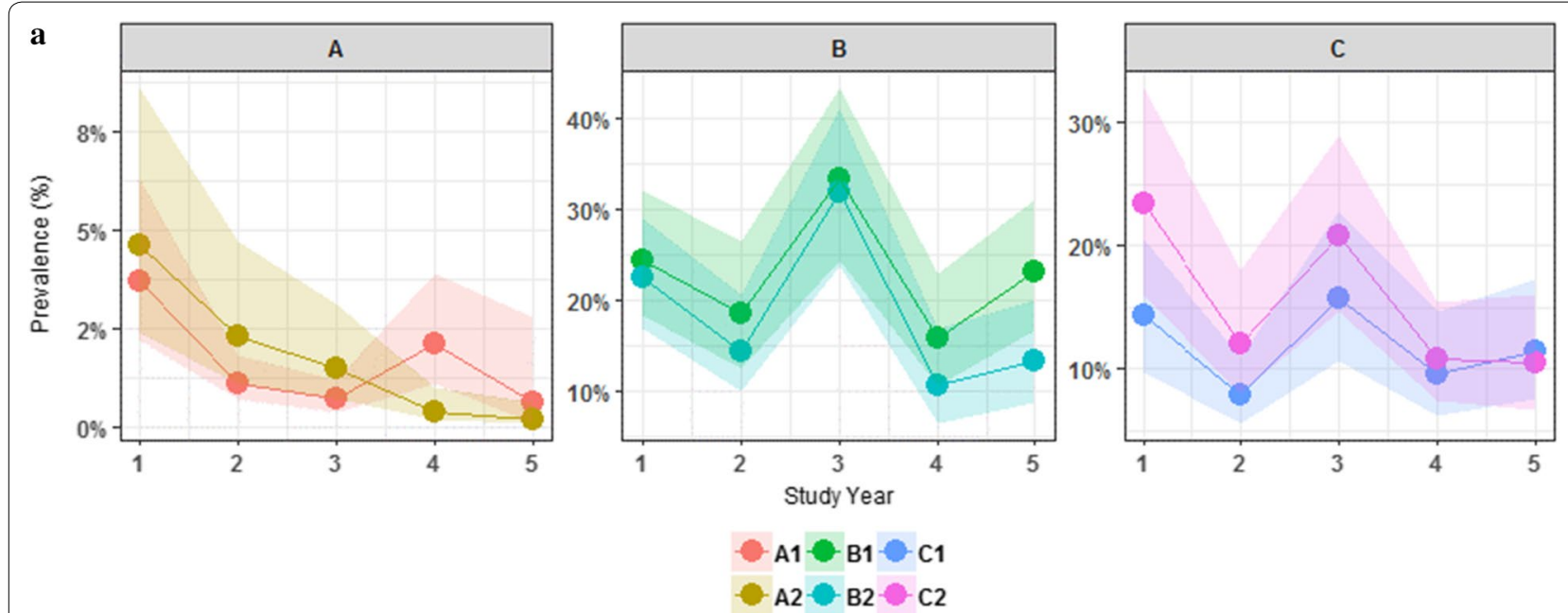

$A 1-B 1-C 1$

$A 2-B 2-C 2$
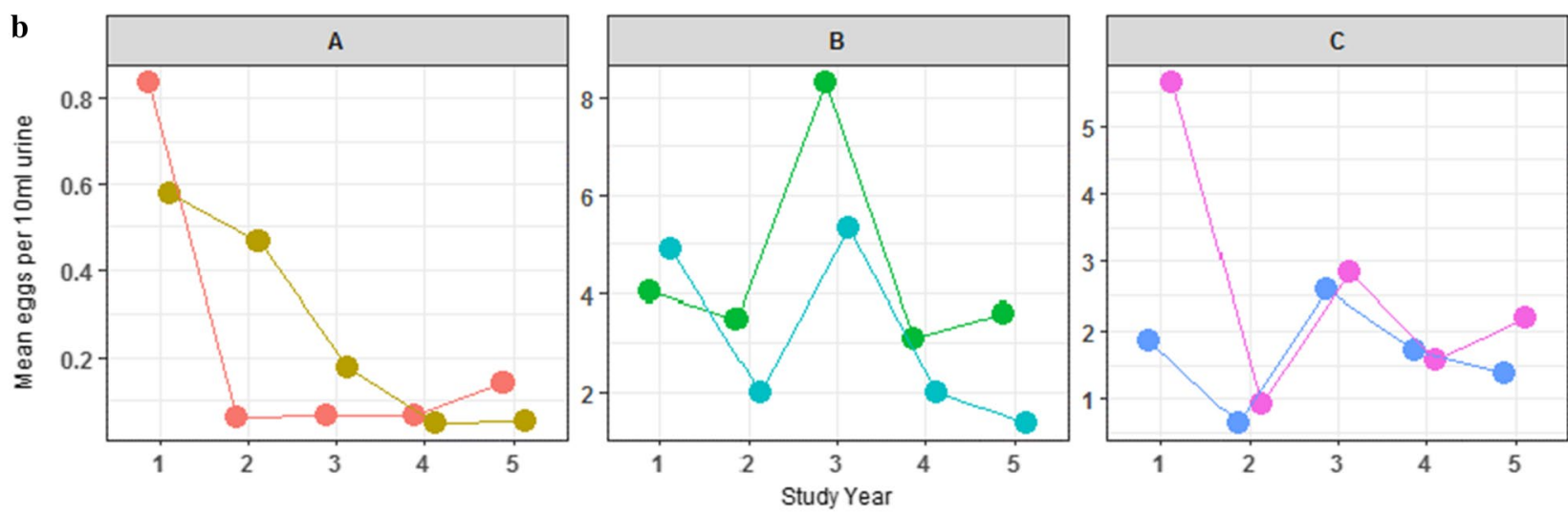

$\mathrm{A} 1-\mathrm{B} 1-\mathrm{C} 1$

$A 2-B 2-C 2$

Fig. 4 Prevalence $\mathbf{a}$ and mean intensity $\mathbf{b}$ of Schistosoma haematobium infection status by study arm over time (9-12 year-olds cross-section only). The change in overall prevalence and mean intensity of S. haematobium at each time point from the start of the project at baseline to the final Year 5 of the study is shown

The communities in Arm A demonstrate a different the pattern of change in prevalence than Arms B and C, which both show an unexpected spike in infection at Year 3. The villages in Arm A are located away from the Niger River, therefore the communities would be in a low transmission zone. It is likely that geographical location of the sites has affected the rate of reinfection, which in turn will influence treatment outcomes.

Although the purpose of the eligibility survey was to ensure a high enough starting prevalence to make comparisons between treatment arms, the eligibility survey had higher infection rates than the baseline parasitological survey. Several villages had high prevalence using Hemastix ${ }^{\circledR}$, when assessed by urine filtration the prevalence was lower than $10 \%$ (Table 5). Some studies have shown that in very low prevalence settings, microhaematuria can be an unstable proxy for urogenital schistosomiasis $[32,33]$. On the other hand, the sensitivity of microscopic egg detection can vary according to intensity of infection, day-to-day variation in egg secretion, time the sample was collected, and number of examined samples [34-38]. Another potential reason for the difference in dipstick and microscopic examination could be the fact that the eligibility survey was 
Table 4 Adjusted odds ratios from GLMM multivariate logistic regression model of S. haematobium infection at Year 5 only $(n=$ 108,231 observations)

\begin{tabular}{|c|c|c|c|c|c|c|}
\hline Study group & Variable & Category & Parameter & Adjusted ORs ${ }^{\mathrm{a}}$ & $95 \% \mathrm{Cl}$ & $P$-values \\
\hline \multirow[t]{5}{*}{ Group A } & \multirow[t]{3}{*}{ Age } & 10 years & -1.09 & 0.34 & $0.11-1.01$ & 0.052 \\
\hline & & 11 years & -1.00 & 0.37 & $0.11-1.20$ & 0.098 \\
\hline & & 12 years & -0.81 & 0.44 & $0.17-1.18$ & 0.104 \\
\hline & Sex & Male & 0.07 & 1.07 & $0.48-2.35$ & 0.871 \\
\hline & Treatment & Biannual treatment & 0.58 & 1.79 & $0.1-33.42$ & 0.698 \\
\hline \multirow[t]{5}{*}{ Group B } & \multirow[t]{3}{*}{ Age } & 10 years & -0.17 & 0.84 & $0.69-1.03$ & 0.090 \\
\hline & & 11 years & -0.28 & 0.75 & $0.61-0.94$ & 0.011 \\
\hline & & 12 years & -0.48 & 0.62 & $0.5-0.77$ & $<0.001$ \\
\hline & Sex & Male & 0.26 & 1.30 & $1.13-1.50$ & $<0.001$ \\
\hline & Treatment & Biannual treatment & -0.84 & 0.43 & $0.2-0.95$ & 0.037 \\
\hline \multirow[t]{5}{*}{ Group C } & \multirow[t]{3}{*}{ Age } & 10 years & 0.03 & 1.03 & $0.83-1.28$ & 0.815 \\
\hline & & 11 years & -0.17 & 0.84 & $0.65-1.08$ & 0.178 \\
\hline & & 12 years & -0.30 & 0.74 & $0.57-0.95$ & 0.019 \\
\hline & Sex & Male & 0.09 & 1.10 & $0.93-1.29$ & 0.272 \\
\hline & Treatment & Biannual treatment & 0 & 1 & $0.43-2.33$ & 0.999 \\
\hline
\end{tabular}

a $95 \% \mathrm{Cls}$ are based on empirical standard errors

Reference groups: Age (9 years); Sex (female); Treatment (annual treatment). Statistically significant $P$-values are indicated in italics

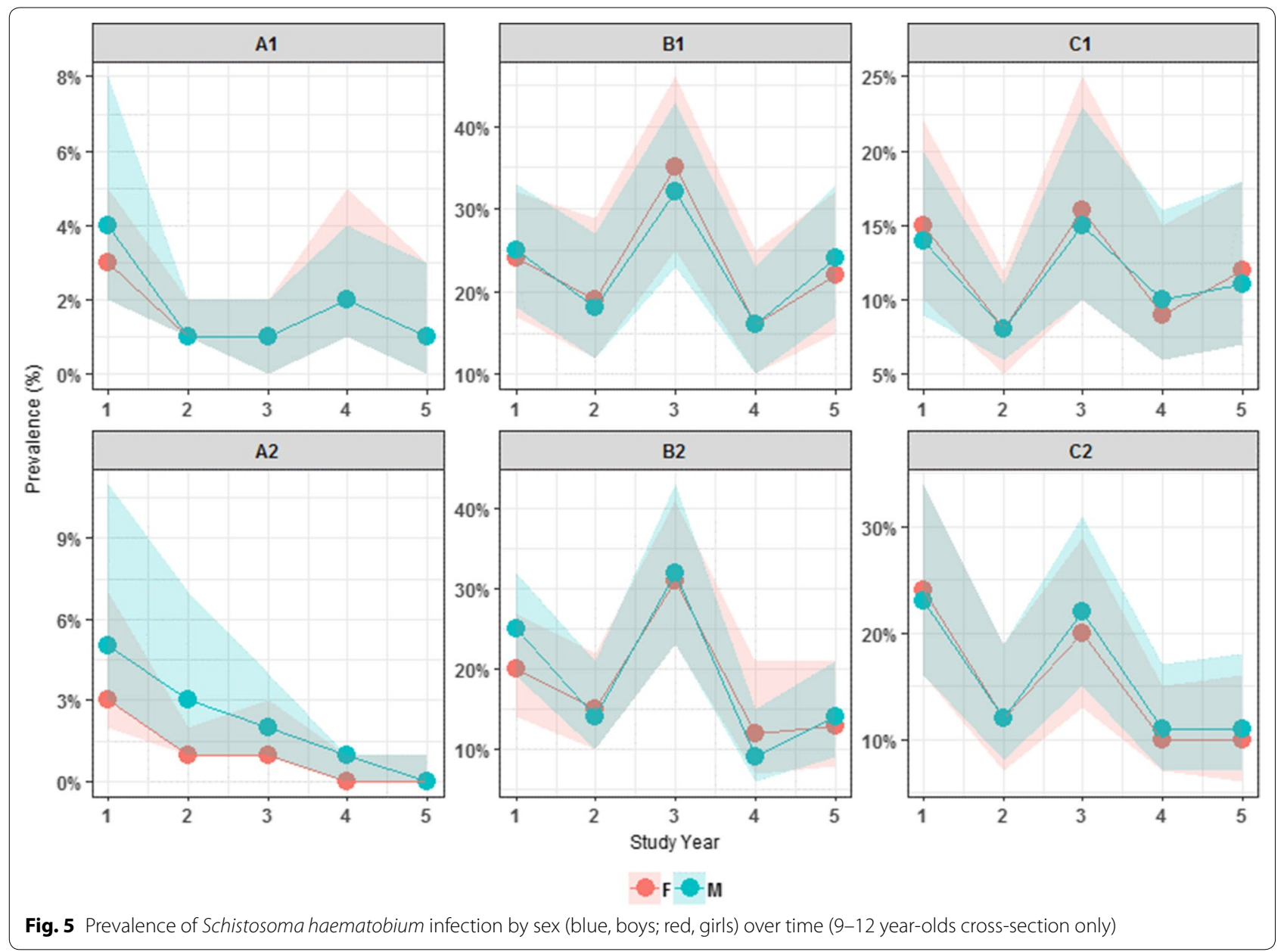



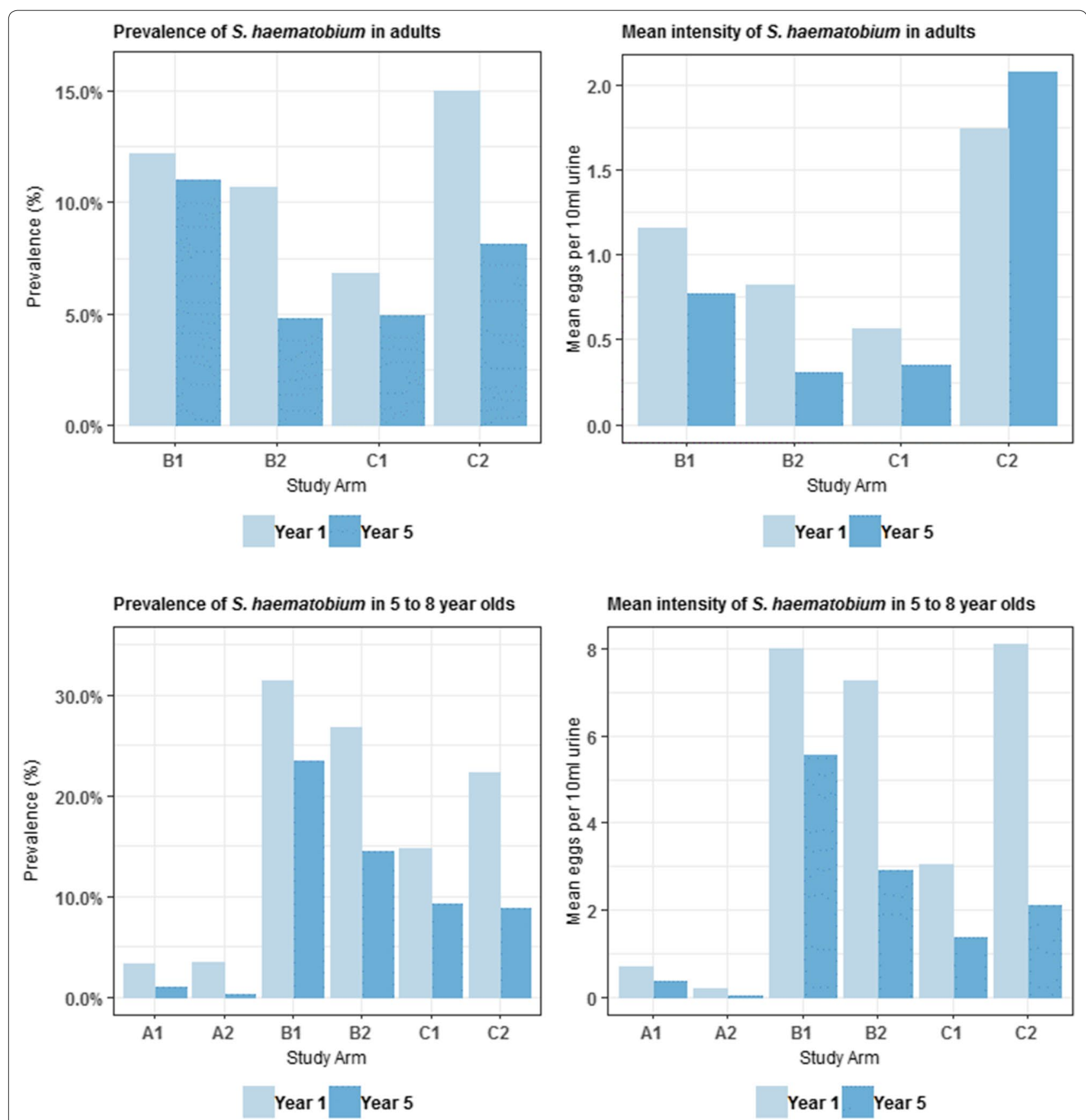

Fig. 6 Prevalence of Schistosoma haematobium infection rates at baseline and Year 5 by study arm (adults and first year student's cross-section only). The change in prevalence and intensity among adults (20-55 years-old) and first year students (5-8 years-old) across all study arms from baseline to Year 5 is shown

carried out in 13-14 year-olds and the parasitology survey among 9-12 year-olds. Some studies have shown that 13-14 year-olds harbour higher infection levels due to them not attending school and therefore more likely to be exposed to infection [39]. Other studies have showed that many cases of urogenital schistosomiasis stay undetected when the examination method is limited to urine filtration [40, 41].

Both age and sex influenced infection where the highest prevalence and intensities of infection occurred in males and among older adolescents, with infection decreasing in adulthood [42, 43]. There was no 
significant difference, however, in response to treatment approach by age and sex.

To evaluate the potential longer-term impact of the programme, we also examined infection in first-year students, who had not received treatment previously as they had only recently started attending school. Schistosoma haematobium infection in this group of children decreased significantly over four rounds of treatment, implying that the level of environmental transmission in these areas could also have been reduced.

Currently the justification for targeting adults in a schistosomiasis control programme is based on the prevalence of infection in SAC. A study in Nigeria, however, has shown that monitoring and evaluation of SAC was not a successful indicator of the burden of infection in adults [44]. Prevalence among adults will likely be driven by many local behavioural and cultural factors, and is, therefore, context specific. This makes it difficult to make a SAC infection threshold for switching to CWT. For this reason, adults were also sampled in this study. The findings here demonstrated that biannual CWT was not significantly more effective at reducing prevalence and intensity of infection. This lack of statistical significance may be attributed to the difference in starting prevalence of the two CWT groups where Group C1 was $14.3 \%$ and $\mathrm{C} 2$ was $23.4 \%$. Indeed, it has been modelled by Turner et al. [16] that the higher the pre-control burden in adults, the larger the benefit of switching to CWT. Interestingly, there was a significant reduction in prevalence of S. haematobium infection among adults even in Group $B$ implying that the rate of transmission in the overall community had been decreased, even where only school children have been treated. These findings of reduction in infection among adults even in SBT communities were also seen in Mozambique [45].

Research in Niger has shown that the full economic delivery cost of SBT in 2005-2006 was \$0.76 and CWT was $\$ 0.46$. Including only the programme costs the figures were $\$ 0.47$ and $\$ 0.41$ respectively [46]. Differences at the sub-district level were more marked. This is partly explained by the fact that a CDD treats 5.8 people for every one treated in school [46]. Given that prevalence decreased among adults in SBT areas and twice-yearly CWT did not have a significantly greater impact on prevalence reduction with respect to annual CWT, these findings have significant logistical and cost-saving implications for a national control programme.

The success of PC programmes may be influenced by a wide range of factors such as initial endemicity and transmission intensities in the local environment, treatment frequency, coverage and compliance, among others [47]. Coverage data among different age groups is an important key determinant for achieving programme targets. Although average treatment coverage in the Niger SCORE programme has been shown to be well above the recommended WHO 75\% threshold, in some areas it was above $100 \%$ and therefore implies an unreliable denominator. This is a common challenge for national control programmes as population census' are often out of date or inaccurate. There may have been other external issues that might have affected the success of the biannual treatment. For example, there may have been a considerable gap between coverage and compliance [48]. Even when the proportion of eligible people who received tablets reach a significant fraction of the target population (coverage), those ingesting all the tablets

Table 5 Prevalence of S. haematobium in western Niger using Haemastix reagent dipsticks (2010-2011)

\begin{tabular}{|c|c|c|c|c|c|c|c|c|}
\hline \multirow[t]{2}{*}{ District } & \multirow[t]{2}{*}{$\begin{array}{l}\text { No. of schools } \\
\text { sampled }\end{array}$} & \multirow[t]{2}{*}{$\begin{array}{l}\text { No. of people } \\
\text { sampled }\end{array}$} & \multirow[t]{2}{*}{$\begin{array}{l}\text { No. of } \\
\text { schools } \\
\text { eligible }\end{array}$} & \multirow[t]{2}{*}{$\begin{array}{l}\text { No. of people sampled } \\
\text { in eligible schools }\end{array}$} & \multicolumn{2}{|c|}{$\begin{array}{l}\text { Method: Dipstick (Eligibility } \\
\text { survey among 13-14 year-olds) }\end{array}$} & \multicolumn{2}{|c|}{$\begin{array}{l}\text { Method: Urine filtration } \\
\text { (Baseline data collection } \\
\text { among 9-12 year-olds) }\end{array}$} \\
\hline & & & & & Prevalence (\%) & $95 \% \mathrm{Cl}$ & Prevalence (\%) & $95 \% \mathrm{Cl}$ \\
\hline CUN5 & 3 & 150 & 1 & 50 & 58.00 & na & 4.00 & na \\
\hline DS1 & 1 & 50 & 1 & 50 & 54.00 & na & 32.00 & na \\
\hline DS3 & 1 & 50 & 1 & 50 & 30.00 & na & 8.00 & na \\
\hline Filingue & 93 & 4528 & 44 & 2141 & 20.10 & $16.71-23.50$ & 5.72 & $2.93-8.52$ \\
\hline Kollo & 87 & 4235 & 52 & 2558 & 42.43 & $36.35-48.51$ & 26.68 & $19.72-33.64$ \\
\hline Loga & 54 & 2641 & 26 & 1281 & 13.75 & $12.03-15.47$ & 1.70 & $0.78-2.62$ \\
\hline Say & 76 & 3568 & 31 & 1501 & 37.19 & $31.79-42.60$ & 13.07 & $7.90-18.24$ \\
\hline Tera & 45 & 2242 & 41 & 2042 & 39.92 & $33.87-45.96$ & 18.79 & $12.51-25.07$ \\
\hline Tillaberi & 32 & 1632 & 28 & 1399 & 38.12 & $27.22-49.03$ & 24.29 & $13.10-35.49$ \\
\hline Total & 392 & 19,096 & 225 & 11,072 & & & & \\
\hline
\end{tabular}

na not applicable 
at the same time (compliance) may be a better indicator of how well PC is being implemented. In this study, SBT was directly observed as the survey teams were present in the schools during the treatment. For CWT, the CDD supervised consumption of the treatment. In addition, frequent migration of people particularly among fishing communities or the nomadic Peule communities, some of whom move between neighbouring countries, remain significant hurdles to the successful implementation of the control programme, particularly in terms of ensuring repeated treatment. Finally, there was an increase in prevalence seen across all Groups in Year 3. This is likely the consequence of severe flooding across the country in 2012, which would have increased water contact as well as affecting the snail population, in addition to subsequent migration.

There were limitations to the study. One was the treatment coverage, discussed above, particularly where adults presented for treatment at the school in the SBT arms. The other limitation was the differential in time periods between MDAs and parasitological surveys, which were not conducted precisely at once and six-monthly timepoints due to the logistical challenge of conducting such large surveys and treatment in such a short time frame. It is not clear whether this contributed to the findings as it was difficult to account for this in the analysis.

The findings here show that with good coverage biannual SBT may be the best strategy for schistosomiasis control in high prevalence areas. In low transmission settings, however, the lack of statistical impact of two rounds of treatment indicate that it may not be possible to break transmission through PC alone and using other strategies such as health education, water, sanitation and hygiene (WaSH), and snail control, should also be considered $[49,50]$.

\section{Conclusions}

The rationale behind biannual treatment was to reduce further the prevalence and intensity of infection, meaning a greater impact on schistosomiasis control. We conclude that twice yearly SBT treatment was effective in reducing $S$. haematobium in high prevalence areas, with reported high treatment coverage. Biannual SBT in low prevalence settings and CWT in high prevalence areas did not have a significant impact on infection levels; therefore, it might not be cost-effective to treat biannually in such areas but focus on more targeted treatment in persistent hot spot areas, where infection remains high despite years of treatment. Finally, there was a significant reduction in prevalence observed in non-targeted age groups in the SBT communities. This suggests that SBT has an impact at reducing transmission potential in the community. The effect this has on adults and other non-targeted age groups should be investigated further as there would be significant cost implications in maintaining SBT strategies only.

\begin{abstract}
Acknowledgements
We are grateful to the members of SCORE secretariat and advisory committee for reviewing our study, their advice, input, and support of our work. We are grateful to the Schistosomiasis Control Initiative for providing praziquantel for the study, through the donation to the Ministry of Health in Niger. We thank the technicians for their support in the field and the laboratory. We are grateful to the health, education, and village authorities of all districts for their contribution. Finally, we thank all the children, parents, teachers, and village leaders who participated in this study.
\end{abstract}

\section{Authors' contributions}

AEP was responsible for the formulation of the overarching research aim of the manuscript. AEP and NAD were responsible for the data curation and analysis. AEP, AG and AF were responsible for the funding acquisition. ZT, BS, IG, $\mathrm{BS}, \mathrm{AGN}, \mathrm{BM}, \mathrm{OA}, \mathrm{HS}, \mathrm{KMH}, \mathrm{RA}$ and $\mathrm{AAH}$ were responsible for the investigation and data collection. AEP, AG and AAH provided the resources for the study and were involved in the overall administration of the project. AEP wrote the paper. All authors read and approved the final manuscript.

\section{Funding}

This research was financially supported by the Bill \& Melinda Gates Foundation through the Schistosomiasis Consortium for Operational Research and Evaluation (SCORE) based at the University of Georgia in Athens, USA, Grant RR374-053/4893196. Praziquantel tablets for schistosomiasis treatment are donated by the Schistosomiasis Control Initiative Foundation (SCIF), UK.

\section{Availability of data and materials}

Data supporting the conclusions of this article are included within the article. The datasets used and analysed during this study are available from the corresponding author upon reasonable request.

\section{Ethics approval and consent to participate}

National and local health, educational and administrative authorities were comprehensively in-formed of the study. Prior to the start of the study in each village, open public meetings were carried out in the local language followed by question and answer sessions with the survey team. The purpose of the study was explained to all schoolchildren, and verbal consent was obtained from the children themselves. It was explained that any child had the chance to withdraw from the study at any point, without any consequence. Written informed consent was also obtained from school headmasters. Due to the high rate of illiteracy among these communities, finger-print consent was obtained from all participants and parents or legal guardians of the children. The study protocol has been approved by the National Ethics Committee in Niger (ref 012/2010/CCNE) and Imperial College Research Ethics Committee (ref ICREC_8_2_2).In addition to these, the University of Georgia institutional review board IRB implemented an administrative human subjects review and issued additional approval 10533-0 for Niger.Informed consent was obtained from parents or legal guardians of all pupils involved in the study and verbal consent was also obtained from all adults included. The purpose of the study was explained to education and health authorities. All results in the datasets were anonymous and coded to prevent association with the individual participant.

\section{Consent for publication}

Not applicable.

\section{Competing interests}

The authors declare that they have no competing interests.

\section{Author details}

${ }^{1}$ London Centre for Neglected Tropical Disease Research (LCNTDR), Department of Infectious Disease Epidemiology, Imperial College London, London, UK. ${ }^{2}$ Aménagement et Lutte (RISEAL NIGER), Réseau International Schistosomiases Environnement, Avenue de l'indépendance, BP. 13724, Niamey, Niger. ${ }^{3}$ Schistosomiasis control Initiative (SCI), Department of Infectious Disease Epidemiology, Imperial College London, London, UK. ${ }^{4}$ Ministère de la Santé, 
Niamey, Niger. ${ }^{5}$ Hôpital National de Niamey rond-point Hôpital, BP 238, Niamey, Niger.

\section{Received: 29 June 2020 Accepted: 21 October 2020}

Published online: 18 November 2020

\section{References}

1. Steinmann P, Keiser J, Bos R, Tanner M, Utzinger J. Schistosomiasis and water resources development: systematic review, meta-analysis, and estimates of people at risk. Lancet Infect Dis. 2006;6:411-25.

2. WHO. Update on the global status of implementation of preventive chemotherapy (PC). Geneva: World Health Organization; 2019. https:// www.who.int/neglected_diseases/preventive_chemotherapy/PC_Updat e.pdf. Accessed 14 Sep 2020.

3. Lai Y-S, Biedermann P, Ekpo UF, Garba A, Mathieu E, Midzi N, et al. Spatial distribution of schistosomiasis and treatment needs in sub-Saharan Africa: a systematic review and geostatistical analysis. Lancet Infect Dis. 2015;15:927-40

4. Clements A, Garba A, Sacko M, Touré S, Dembelé R, Landouré A, et al. Mapping the probability of schistosomiasis and associated uncertainty. West Africa Emerg Infect Dis. 2008;14:1629-32.

5. Garba A, Touré S, Dembelé R, Bosque-Oliva E, Fenwick A. Implementation of national schistosomiasis control programmes in West Africa. Trends Parasitol. 2006;22:322-6.

6. WHO. Preventive chemotherapy in human helminthiasis: coordinated use of anthelminthic drugs in control interventions: a manual for health professionals and programme managers. Geneva: World Health Organization; 2006.

7. Gabrielli AF, Toure S, Sellin B, Sellin E, Ky C, Ouedraogo H, et al. A combined school- and community-based campaign targeting all school-age children of Burkina Faso against schistosomiasis and soil-transmitted helminthiasis: performance, financial costs and implications for sustainability. Acta Trop. 2006;99:234-42.

8. Salam RA, Maredia H, Das JK, Lassi ZS, Bhutta ZA. Community-based interventions for the prevention and control of helminthic neglected tropical diseases. Infect Dis Poverty. 2014;3:23.

9. Hanson C, Weaver A, Zoerhoff KL, Kabore A, Linehan M, Doherty A, et al. Integrated implementation of programs targeting neglected tropical diseases through preventive chemotherapy: identifying best practices to roll out programs at national scale. Am J Trop Med Hyg. 2012;86:508-13.

10. Toor J, Turner HC, Truscott JE, Werkman M, Phillips AE, Alsallaq R, et al. The design of schistosomiasis monitoring and evaluation programmes: the importance of collecting adult data to inform treatment strategies for Schistosoma mansoni. PLoS Negl Trop Dis. 2018;12:e0006717.

11. Anderson RM, Turner HC, Farrell SH, Yang J, Truscott JE. What is required in terms of mass drug administration to interrupt the transmission of schistosome parasites in regions of endemic infection? Parasit Vectors. 2015;8:553.

12. Anderson RM, Turner HC, Farrell SH, Truscott JE. Studies of the transmission dynamics, mathematical model development, and the control of schistosome parasites by mass drug administration in human communities. Adv Parasitol. 2016:94:199-246.

13. Njenga SM, Mwandawiro CS, Muniu E, Mwanje MT, Haji FM, Bockarie MJ. Adult population as potential reservoir of NTD infections in rural villages of Kwale district. Coastal Kenya. 2011;4:175

14. Lo NC, Bogoch II, Blackburn BG, Raso G, N'Goran EK, Coulibaly JT, et al. Comparison of community-wide, integrated mass drug administration strategies for schistosomiasis and soil-transmitted helminthiasis: a costeffectiveness modelling study. Lancet Glob Health. 2015;3:629-38.

15. Gurarie D, Yoon N, Li E, Ndeffo-Mbah M, Durham D, Phillips AE, et al. Modelling control of Schistosoma haematobium infection: predictions of the long-term impact of mass drug administration in Africa. Parasit Vectors. 2015:8:529.

16. Turner HC, Truscott JE, Bettis AA, Farrell SH, Deol AK, Whitton JM, et al. Evaluating the variation in the projected benefit of community-wide mass treatment for schistosomiasis: implications for future economic evaluations. Parasit Vectors. 2017;10:213
17. Massa K, Magnussen P, Sheshe A, Ntakamulenga R, Ndawi B, Olsen A. The effect of the community-directed treatment approach versus the school-based treatment approach on the prevalence and intensity of schistosomiasis and soil-transmitted helminthiasis among schoolchildren in Tanzania. Trans R Soc Trop Med Hyg. 2009;103:31-7.

18. Onkanga IO, Mwinzi PNM, Muchiri G, Andiego K, Omedo M, Karanja DMS, et al. Impact of two rounds of praziquantel mass drug administration on Schistosoma mansoni infection prevalence and intensity: a comparison between community wide treatment and school-based treatment in western Kenya. Int J Parasitol. 2016;46:439-45.

19. King $\mathrm{CH}$, Olbrych SK, Soon M, Singer ME, Carter J, Colley DG. Utility of repeated praziquentel dosing in the treatment of schistosomiasis in high-risk communities in Africa: a systematic review. PLoS Negl Trop Dis. 2011:5:e1321.

20. Toure S, Zhang Y, Bosque-Oliva E, Ky C, Ouedraogo A, Koukounari A, et al. Two-year impact of single praziquantel treatment on infection in the national control programme on schistosomiasis in Burkina Faso. Bull World Health Organ. 2008:86:780-7.

21. Ezeamama AE, He CL, Shen Y, Yin XP, Binder SC, Campbell CH, et al. Gaining and sustaining schistosomiasis control: study protocol and baseline data prior to different treatment strategies in five African countries. BMC Infect Dis. 2016;16:229.

22. Colley DG. Morbidity control of schistosomiasis by mass drug administration: How can we do it best and what will it take to move on to elimination? Trop Med Health. 2014;42(Suppl. 2):25-32.

23. Assaré RK, Knopp S, N'Guessan NA, Yapi A, Tian-Bi YN, Yao PK, et al. Sustaining control of schistosomiasis mansoni in moderate endemicity areas in western Côte d'Ivoire: a SCORE study protocol. BMC Public Health. 2014;14:1290.

24. King CH, Bertsch D. Meta-analysis of urine heme dipstick diagnosis of Schistosoma haematobium infection, including low-prevalence and previously-treated populations. PLoS Negl Trop Dis. 2013;7:e2431.

25. United Nations Development Programme. Human Development Report 2016; 2017, 286 pp. https://hdr.undp.org/sites/default/files/2016_human _development_report.pdf.

26. WHO/UNICEF Joint Monitoring Programme for Water Supply and Sanitation (JMP). Progress on household drinking water, sanitation, and hygiene, 2000-2017. https://washdata.org/sites/default/files/documents/ reports/2019-07/jmp-2019-wash-households.pdf Accessed 14 Sep 2020.

27. WHO/UNICEF Joint Monitoring Programme (JMP) for water supply and sanitation. Progress on sanitation and drinking water-2015 update and MDG assessment, 90 pp. https://www.unicef.org/publications/index _82419.html Accessed 14 Sep 2020

28. African Ministers' Council on Water (AMCOW), et al. Getting Africa on Track to Meet the MDGs on Water and Sanitation; 2006. https://docum ents.worldbank.org/en/publication/documents-reports/documentde tail/894781468192233997/getting-africa-on-track-to-meet-the-mdgson-water-and-sanitation-a-status-review-of-sixteen-african-countries Accessed 14 Sep 2020

29. Plouvier S, Leroy JC, Colette J. A propos d'une technique simple de filtration des urines dans le diagnostic de la bilharziose urinaire en enquête de masse. Med Trop. 1975;35:229-30.

30. Montresor A, Engels D, Ramsan M, Foum A, Savioli L. Field test of the 'dose pole' for praziquantel in Zanzibar. Trans R Soc Trop Med Hyg. 2002;96:323-4.

31. R Core Team. R: A language and environment for statistical computing. Vienna: R Foundation for Statistical Computing; 2015. https://www.Rproject.org. Accessed Apr 2016.

32. Krauth SJ, Greter H, Stete K, Coulibaly JT, Traoré SI, Ngandolo BN, Achi LY, Zinsstag J, N'Goran EK, Utzinger J. All that is blood is not schistosomiasis: experiences with reagent strip testing for urogenital schistosomiasis with special consideration to very-low prevalence settings. Parasit Vectors. 2015;8:584

33. Ochodo EA, Gopalakrishna G, Spek B, Reitsma JB, van Lieshout L, Polman $\mathrm{K}$, et al. Circulating antigen tests and urine reagent strips for diagnosis of active schistosomiasis in endemic areas. Cochrane Database Syst Rev. 2015;3:CD009579.

34. Le L, Hsieh MH. Diagnosing urogenital schistosomiasis: dealing with diminishing returns. Trends Parasitol. 2017;33:378-87. 
35. Chandiwana SK. Community water-contact patterns and the transmission of Schistosoma haematobium in the Highveld region of Zimbabwe. Soc Sci Med. 1987;25:495-505.

36. Kabatereine NB, Kemijumbi J, Ouma JH, Kariuki HC, Richter J, Kadzo H, et al. Epidemiology and morbidity of Schistosoma mansoni infection in a fishing community along Lake Albert in Uganda. Trans R Soc Trop Med Hyg. 2004;98:711-8.

37. Feldmeier $\mathrm{H}$, Poggensee $\mathrm{G}$. Diagnostic techniques in schistosomiasis control. A review. Acta Trop. 1993:52:205-20.

38. McCarthy JS, Lustigman S, Yang GJ, Barakat RM, García HH, Sripa B, et al. A research agenda for helminth diseases of humans: diagnostics for control and elimination programmes. PLoS Negl Trop Dis. 2012;6:e1601.

39. Woolhouse MEJ, Mutapi F, Ndhlovu PD, Chandiwana SK, Hagan P. Exposure, infection and immune responses to Schistosoma haematobium in young children. Parasitology. 2000;120:37-44.

40. Poggensee G, Kiwelu I, Saria M, Richter J, Krantz I, Feldmeier H. Schistosomiasis of the lower reproductive tract without egg excretion in urine. Am J Trop Med Hyg. 1998;59:782-3.

41. Hegertun IE, SulheimGundersen KM, Kleppa E, Zulu SG, Gundersen SG, Taylor M, Kvalsig JD, Kjetland EF. Schistosoma haematobium as a common cause of genital morbidity in girls: a cross-sectional study of children in South Africa. PLoS Negl Trop Dis. 2013;7:e2104.

42. Hagan P, Chandiwana S, Ndhlovu P, Woolhouse MEJ, Dessein A. The epidemiology, immunology and morbidity of Schistosoma haematobium infections in diverse communities in Zimbabwe. I: The study design. Trop Geogr Med. 1994;46:227-32.

43. Vennervald BJ, Ouma JH, Butterworth AE. Morbidity in schistosomiasis: assessment. Parasitol Today. 1998;14:385-90.

44. Evans DS, King JD, Eigege A, Umaru J, Adamani W, Alphonsus K, et al. Assessing the $\mathrm{WHO} 50 \%$ prevalence threshold in school-aged children as indication for treatment of urogenital schistosomiasis in adults in central Nigeria. Am J Trop Med Hyg. 2013;88:441-5.

45. Phillips AE, Gazzinelli-Guimaraes PH, Aurelio HO, Ferro J, Rassul N, Clements $\mathrm{M}$, King $\mathrm{CH}$, et al. Assessing the benefits of five years of different approaches to treatment of urogenital schistosomiasis: a SCORE project in Northern Mozambique. PLoS Negl Trop Dis. 11(12): e0006061.

46. Leslie J, Garba A, Oliva EB, Barkire A, Tinni AA, Djibo A, Mounkaila I, Fenwick A. Schistosomiasis and soil-transmitted helminth control in Niger: cost-effectiveness of school-based and community distributed mass drug administration. PLoS Negl Trop Dis. 2011;5:e1326.

47. Prichard RK, Basáñez MG, Boatin BA, McCarthy JS, García HH, Yang GJ, et al. A research agenda for helminth diseases of humans: intervention for control and elimination. PLoS Negl Trop Dis. 2012;6:e1549.

48. Shuford KV, Turner HC, Anderson RM. Compliance with anthelmintic treatment in the neglected tropical disease control programmes: a systematic review. Parasit Vectors. 2016;9:29.

49. WHO. Meeting of the International task force for disease eradication, April 2012. Wkly Epidemiol Rec. 2012;87:305-9.

50. Stothard JR, Campbell SJ, Osei-Atweneboana MY, Durant T, Stanton MC, Biritwum N-K, et al. Towards interruption of schistosomiasis transmission in sub-Saharan Africa: developing an appropriate environmental surveillance framework to guide and to support 'end game' interventions. Infect Dis Poverty. 2017;6:10

\section{Publisher's Note}

Springer Nature remains neutral with regard to jurisdictional claims in published maps and institutional affiliations.
Ready to submit your research? Choose BMC and benefit from:

- fast, convenient online submission

- thorough peer review by experienced researchers in your field

- rapid publication on acceptance

- support for research data, including large and complex data types

- gold Open Access which fosters wider collaboration and increased citations

- maximum visibility for your research: over $100 \mathrm{M}$ website views per year

At BMC, research is always in progress.

Learn more biomedcentral.com/submissions 